\title{
MODELO DE BANCO DE DADOS PARA GERENCIAMENTO DOS TESTES DE CONTROLE DE QUALIDADE DOS EQUIPAMENTOS DE RADIOLOGIA CONVENCIONAL DO HOSPITAL UNIVERSITÁRIO CLEMENTINO FRAGA FILHO*
}

\author{
Rildo Santos Ribeiro ${ }^{1}$, Nelson Fraga do Couto ${ }^{1}$, Antonio Carlos Pires Carvalho², Ana Cecília \\ Pedrosa de Azevedo 3
}

\begin{abstract}
Resumo Foi elaborado um sistema para gerenciamento informatizado dos testes de controle de qualidade exigidos pela Portaria 453/98 da Secretaria de Vigilância Sanitária do Ministério da Saúde, visando otimizar sua execução, armazenamento e interpretação. Os testes de controle de qualidade são parte do programa de garantia de qualidade que deve ser implantado em toda instalação de radiodiagnóstico médico e odontológico. Para isso, foi elaborado um banco de dados utilizando o programa Microsoft Access ${ }^{\circledR}$, permitindo comunicação direta com o sistema informatizado de gerenciamento das manutenções dos equipamentos de raios $\mathrm{X}$, em implantação no Hospital Universitário Clementino Fraga Filho da Universidade Federal do Rio de Janeiro. Unitermos: Sistemas de gerenciamento de base de dados; Controle de qualidade; Radiografia - instrumentação.
\end{abstract}

Abstract Model database used for quality control management of conventional x-ray equipment at Hospital Universitário Clementino Fraga Filho.

A computerized system to manage quality control testing and general $\mathrm{X}$-ray equipment maintenance was developed in accordance with the requirements of Brazilian legislation (Ministerial Order No. 453/98). Quality control tests are part of a quality assurance program that should be implemented in all medical and odontological radiodiagnosis institutions. A Microsoft Access ${ }^{\circledR}$ database was developed to allow a direct link with the maintenance system that is being implemented at the “Hospital Universitário Clementino Fraga Filho - Universidade Federal do Rio de Janeiro", Rio de Janeiro, Brazil.

Key words: Database management systems; Quality control; Radiography - instrumentation.

\section{INTRODUÇÃO}

O uso das radiações ionizantes na prática da medicina é justificável, frente aos benefícios gerados para os pacientes em seus diagnósticos e tratamento. Porém, os riscos associados à sua utilização não devem ser desprezados, mas tratados com muita seriedade, riscos estes que decorrem, muitas vezes, da falta de treinamento adequado na utilização de equipamentos, como, também, na falta de programas de

* Trabalho realizado no Departamento de Radiologia da Faculdade de Medicina e no Hospital Universitário Clementino Fraga Filho (HUCFF), ambos da Universidade Federal do Rio de Janeiro (UFRJ), Rio de Janeiro, RJ.

1. Alunos do Programa de Pós-Graduação em Radiologia da Faculdade de Medicina da UFRJ.

2. Professor Adjunto do Departamento de Radiologia da Faculdade de Medicina da UFRJ.

3. Fiocruz, Escola Nacional de Saúde Pública - CESTEH, Física da Faculdade de Medicina da UFRJ.

Endereço para correspondência: Prof. Dr. Antonio Carlos Pires Carvalho. Rua José Higino, 290, ap. 401, Tijuca. Rio de Janeiro, RJ, 20520-200. E-mail: acpcrj@ufrj.br.

Recebido para publicação em 19/3/2003. Aceito, após revisão, em 19/5/2003. manutenção desses equipamentos e, ainda, da inadequada utilização de equipamentos individuais de radioproteção.

Conforme a Portaria 453/98 da Secretaria de Vigilância Sanitária do Ministério da Saúde (SVS/MS) ${ }^{(\mathbf{1})}$, é obrigatório o cumprimento de normas para funcionamento dos serviços de radiodiagnóstico médico e odontológico, com o objetivo de melhorar a qualidade dos exames realizados, aumentando a confiabilidade dos diagnósticos, e, ainda, promovendo a segurança para funcionários e pacientes.

Com tais propósitos verifica-se, nos itens 3.55 e 3.25-e dessa Portaria, respectivamente:

- Que os titulares do Serviço devem implementar um programa de garantia de qualidade, integrante do programa de proteção radiológica.

- Que sejam tomadas todas as medidas necessárias para evitar falhas e erros, incluindo a implementação de procedimentos adequados de calibração, controle de qualidade e operação dos equipamentos de raios $\mathrm{X}$.

Os primeiros equipamentos utilizados para gerar os raios $\mathrm{X}$ úteis à medicina utilizavam tensões baixas, o que resultava no grande tempo de exposição, uma vez que os fótons possuíam baixa energia e, com isso, grande parte era absorvida pelos tecidos do paciente ${ }^{(2)}$.

Hoje, graças ao avanço tecnológico, contamos com equipamentos com ampla escala de tensão, para ser regulada de acordo com a espessura da região a ser radiografada. Quanto maior a espessura, maior a energia para que os fótons atravessem o paciente e impressionem o filme radiográfico. Mesmo assim, grande quantidade de fótons é absorvida pela pele, e a razão entre a dose de radiação de saída e a radiação de entrada na região da pele do paciente que será radiografada fica em torno de 0,01 ou menos, o que é chamado de relação de transmissão. Assim, um dos principais objetivos é o de aumentar esta relação. 
Alguns fatores contribuem diretamente para a melhoria da relação de transmissão, como, por exemplo, desalinhamento vertical do feixe principal de raios $\mathrm{X}$ em relação ao plano do exame, flutuação da tensão de alimentação do tubo de raios $\mathrm{X}$, filtração da janela do tubo e de partes da carcaça $^{(3)}$, colimação do campo de radiação ${ }^{(4)}$.

Dependendo da dimensão dos erros dos parâmetros citados acima, tem-se a inutilização do exame, o que acarreta sua repetição, acumulando gastos desnecessários para o serviço de radiodiagnóstico e, ainda, exposição desnecessária do paciente à radiação ionizante ${ }^{(4)}$.

Contudo, ainda encontramos, na radiografia convencional, um método barato e eficiente quando ela é usada com responsabilidade, constituindo-se na base do diagnóstico por imagem, muito embora encontremos outros métodos de obtenção de imagem ou, até mesmo, outros métodos de diagnóstico ${ }^{(5)}$. Dessa forma, há de se garantir a qualidade dos serviços de radiodiagnóstico, bem como a padronização dos requisitos de proteção radiológica ${ }^{(6)}$.

A Portaria ainda regulamenta, em seus itens 4.44 e 4.45 , as condições ideais de funcionamento de todos os equipamentos de raios $\mathrm{X}$, que devem passar regularmente por testes de verificação de desempenho, os quais são divididos em quatro grupos, por intervalo de tempo. São eles: testes bianuais, anuais, semestrais e semanais. Tais verificações visam melhorar a qualidade da imagem e garantir a eliminação de toda a radiação que não contribui para a formação de imagem útil ${ }^{(\mathbf{6})}$.

Alguns autores verificam, ainda, que não basta testar os equipamentos de raios $\mathrm{X}^{(\mathbf{7}, \mathbf{8})}$. É de grande importância a qualidade dos equipamentos que auxiliam no diagnóstico, como os negatoscópios. Mesmo dispondo de um aparelho de raios $\mathrm{X}$ dentro dos padrões técnicos de utilização, o paciente pode sofrer com um diagnóstico incorreto, fruto da não observação de detalhes importantes devida à inadequada iluminação do filme durante a interpretação do resultado.

Uma importante regulamentação é a que determina que todos os dados referentes a esse controle de qualidade sejam coletados e armazenados para futuras consultas. Dessa forma, faz-se necessário um sis- tema de armazenamento e controle de dados padronizado e eficiente, tendo em vista a quantidade de dados referente aos testes de todos os equipamentos. Este fato direciona para o controle informatizado de todos os dados dos testes de controle de qualidade.

Alguns autores ${ }^{(\mathbf{9 - 1 3 )}}$ acreditam na importância da utilização da informática na medicina, o que pode gerar controle e uniformização de atividades, diminuição de custos e rapidez de acesso às informações.

Várias experiências foram bem sucedidas com o uso da informática dentro da radiologia. Geraldeli et al. ${ }^{(\mathbf{1 4})}$ criaram um banco dados com o objetivo de digitalizar imagens radiográficas, salvando estas da deterioração e, ainda, facilitando sua manipulação. Já Oliveira et al. ${ }^{(\mathbf{1 5})}$ criaram um banco de dados para monitoração ocupacional dos trabalhadores expostos à radiação no Hospital Universitário Clementino Fraga Filho da Universidade Federal do Rio de Janeiro (HUCFF-UFRJ), o que permitia o controle de todas as informações referentes aos funcionários, controlados por dosímetros.

O objetivo deste trabalho é apresentar um modelo de banco de dados para gerenciar os testes de controle de qualidade dos equipamentos convencionais do Serviço de Radiodiagnóstico do HUCFF-UFRJ.

\section{MATERIAIS E MÉTODOS}

Equipamento utilizado - Foi utilizado um computador comum do tipo PC e programa de gerenciamento de banco de dados Microsoft Access ${ }^{\circledR}$, com plataforma Microsoft Windows $\mathrm{ME}^{\circledR}$.

Levantamento de informações - Foram seguidas algumas etapas para obtenção de informações relevantes à produção do banco de dados, que foram desde observações sobre a rotina de funcionamento das salas de exames, passando por entrevistas com técnicos e médicos, até o estudo acerca dos diferentes testes de controle de qualidade e seus protocolos.

\section{RESULTADOS}

O banco de dados foi elaborado atendendo às exigências da Portaria 453/98 com relação aos testes de controle de qualidade (TCQ). Existe, ainda, a possibilidade de intercâmbio das informações com o setor responsável pela manutenção. $\mathrm{O}$ banco de dados contém um cadastro completo de todos os equipamentos de raios $\mathrm{X}$ pertencentes ao serviço de radiodiagnóstico, o que facilita, no caso de alguns testes, saber detalhes sobre os equipamentos.

A Figura 1 mostra a tela do "menu" com os itens específicos da manutenção, como Almoxarifado e Programa de Manutenção.

Escolhendo o item Programa de Garantia de Qualidade, o usuário poderá entrar nos diferentes TCQ, listados em ordem alfabética, conforme mostra a Figura 2.

Na Figura 3 temos o primeiro teste listado, que trata da camada semi-redutora (item 4.45 b iii da Portaria 453/98). Neste formulário é possível armazenar os dados referentes ao teste, seguindo o protocolo estabelecido, além de buscar e comparar valores de testes realizados anteriormente.

Para o teste de coincidência entre os campos de radiação e luminoso temos o formulário da Figura 4, que permite, ainda, armazenar os dados referentes ao teste de alinhamento do feixe principal de radiação em relação ao eixo ortogonal do plano do exame. Temos os dois testes num só formulário, uma vez que os objetos de teste devem ser utilizados ao mesmo tempo.

Para o item 4.45 b i, referente à necessidade de execução anual do teste de exatidão do indicador de tensão do tubo, temos o formulário da Figura 5, que permite realizar a coleta de dados sobre a reprodutibilidade dos valores de tensão no tubo.

Para aferir a exatidão do tempo de exposição, conforme o item 4.45 b ii, temos o formulário da Figura 6, o qual avalia, ainda, a constância do sistema de controle automático de exposição (AEC - "automatic exposure control"), em conformidade com o item 4.45 b viii (Figura 7).

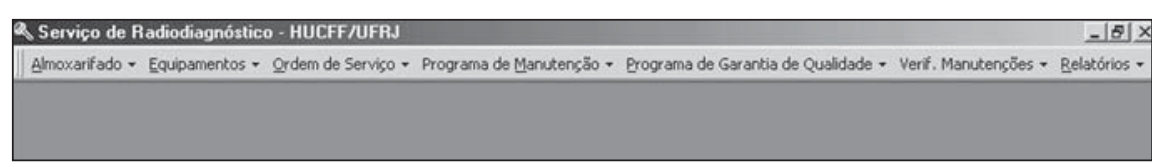

Figura 1. "Menu" principal. 


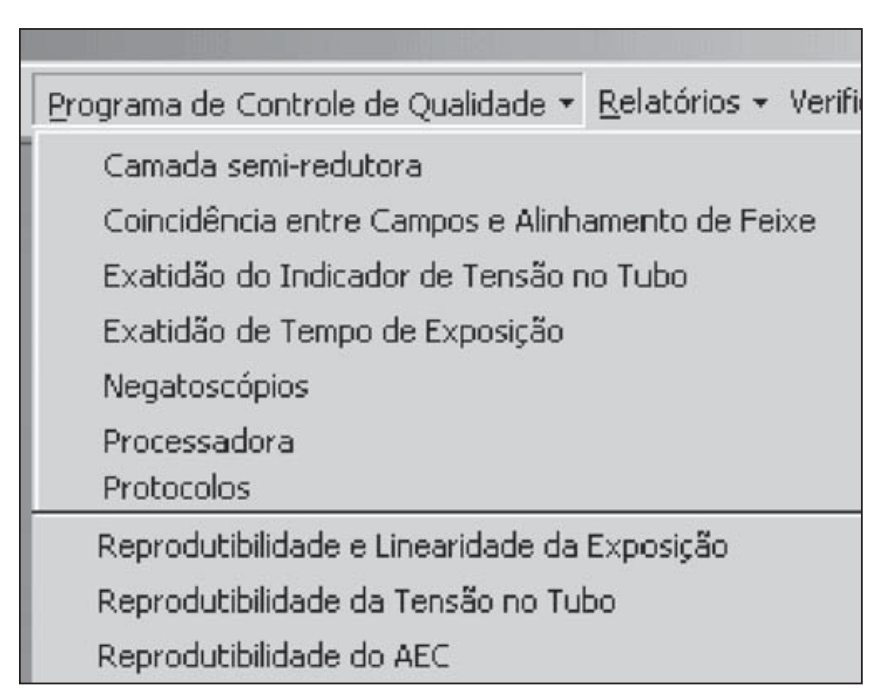

Figura 2. Relação dos TCQ.

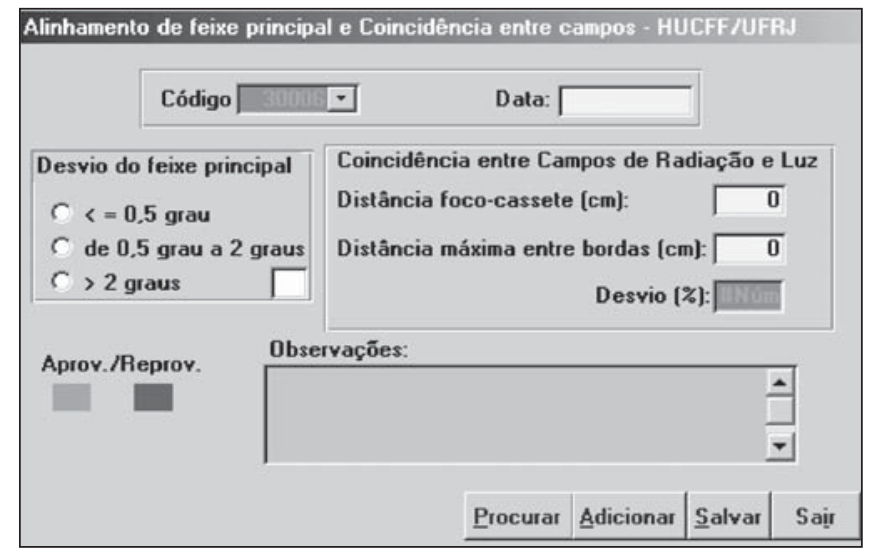

Figura 4. Testes de coincidência entre campos luminoso e de radiação e alinhamento do feixe principal.

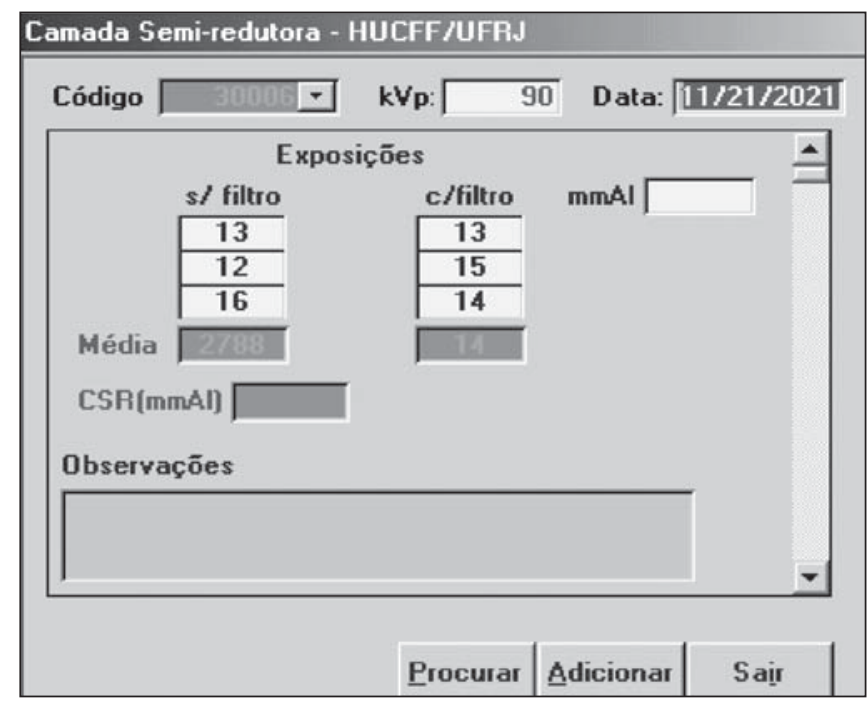

Figura 3. Teste da camada semi-redutora.

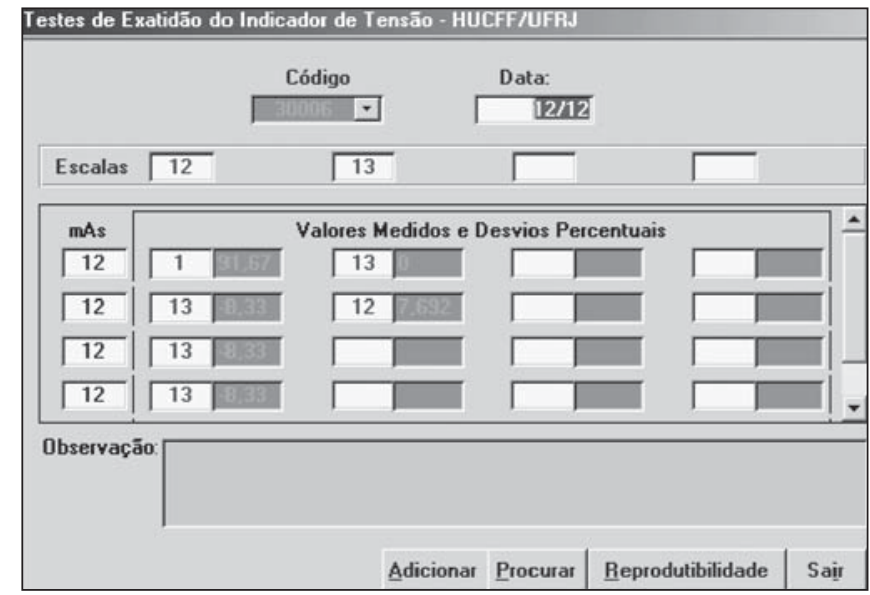

Figura 5. Teste de exatidão da tensão no tubo de raios $X$.

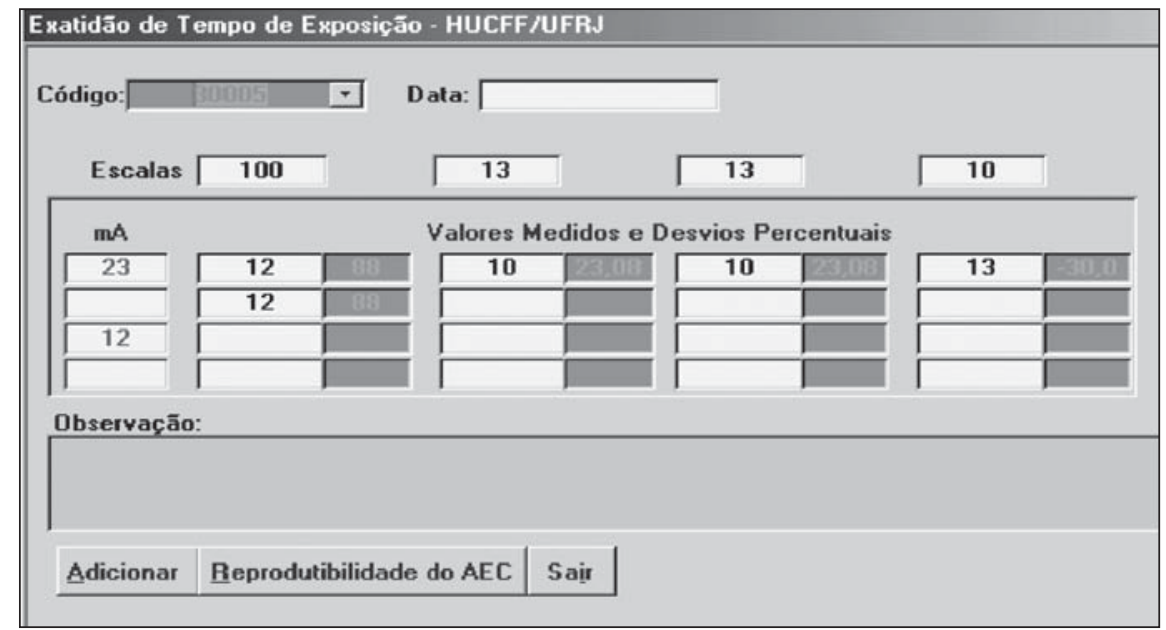

Figura 6. Teste de exatidão do tempo de exposição.

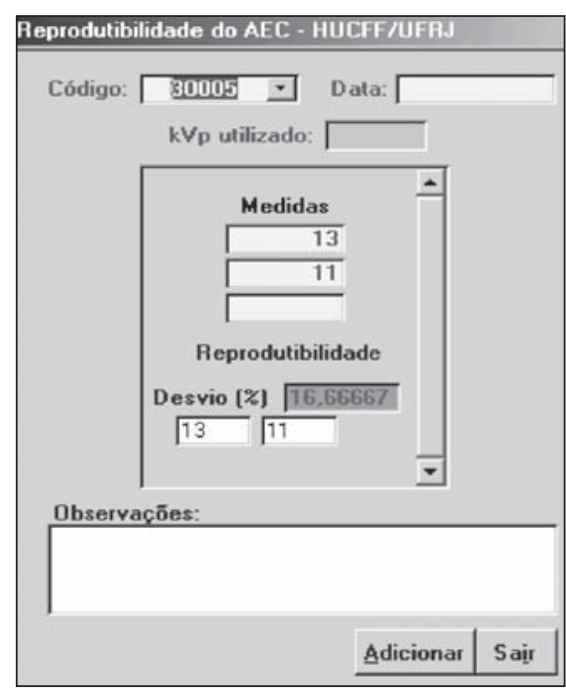

Figura 7. Teste de reprodutibilidade do AEC. 
O formulário da Figura 8 encontra-se baseado no item $4.45 \mathrm{c}$ vi, referindo-se às condições de luminosidade e homogeneidade dos negatoscópios do serviço de radiodiagnóstico.

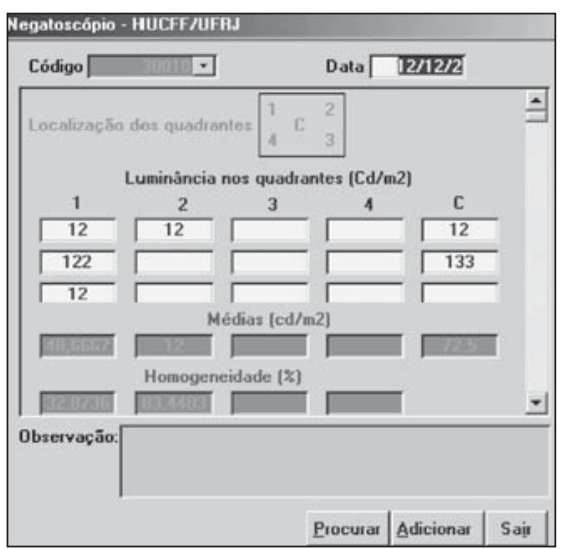

Figura 8. Teste de condições dos negatoscópios.

De acordo com o item $4.45 \mathrm{~d}$ iii, temos o formulário relativo ao teste do sistema de processamento, levantando valores sensitométricos, $\mathrm{pH}$ das substâncias químicas envolvidas na revelação dos filmes radiográficos e suas respectivas temperaturas (Figura 9).

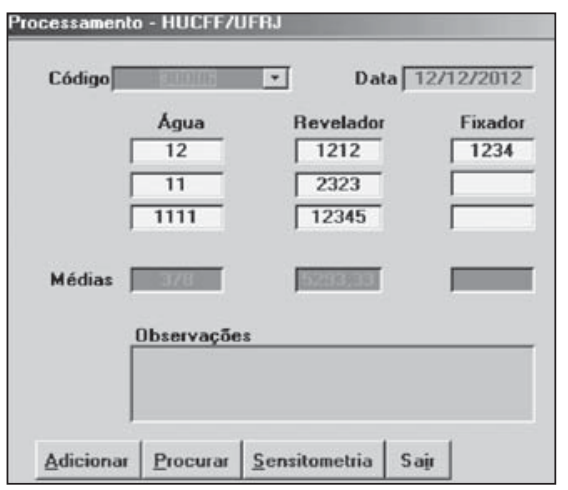

Figura 9. Teste do sistema de processamento.

Em conformidade com os itens $4.45 \mathrm{~b}$ vi e vii, temos o formulário mostrado na Figura 10, o qual armazena e calcula valores dos testes de linearidade e reprodutibilidade da taxa de kerma no ar.

O formulário da Figura 11 refere-se ao teste de reprodutibilidade da tensão no tubo de raios $\mathrm{X}$, o qual pode, também, ser acessado através do botão "Reprodutibilidade", que se encontra no formulário da Figura 6, referente ao teste de exatidão da tensão no tubo de raios X.

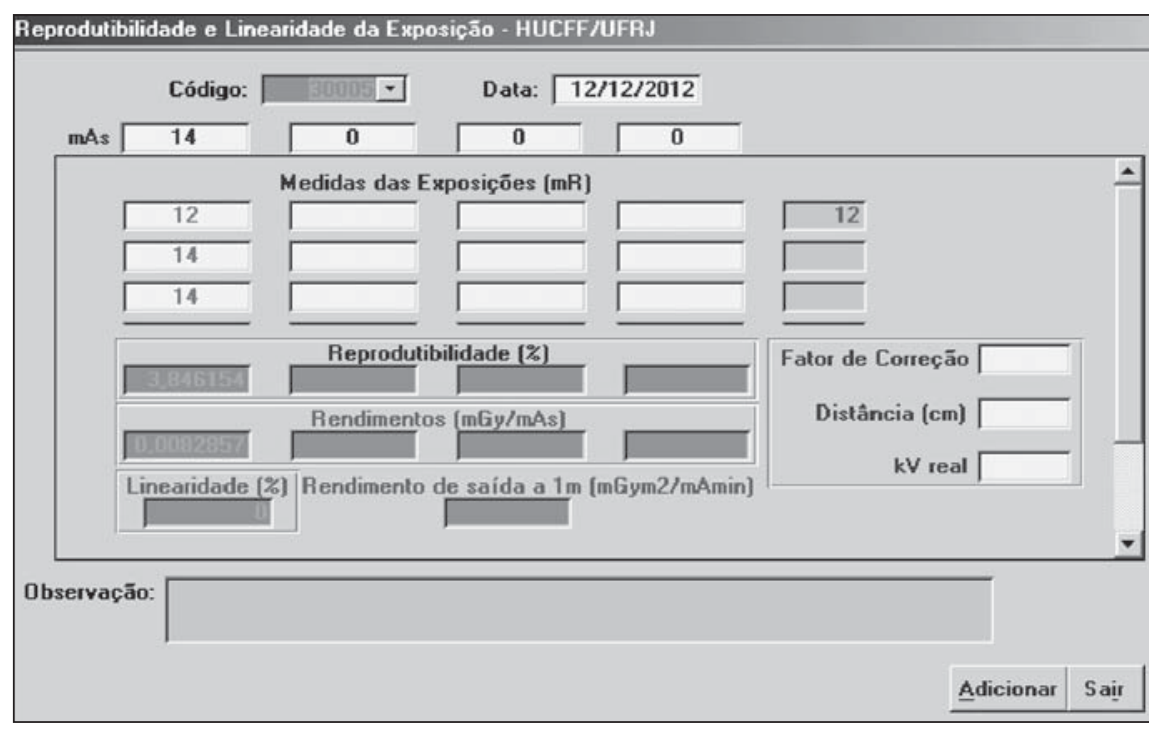

Figura 10. Teste de linearidade e reprodutibilidade da exposição da taxa de kerma no ar.

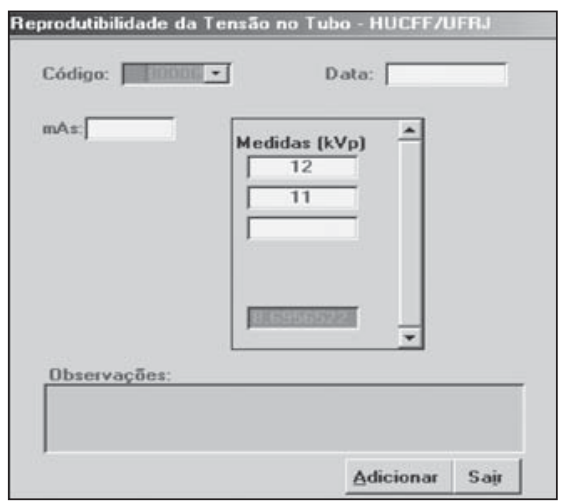

Figura 11. Teste da reprodutibilidade da tensão no tubo.

O banco de dados ainda permite ao usuário acessar, através do formulário da Figura 12, os protocolos dos TCQ e, por meio deles, abrir os respectivos formulários de entrada de dados utilizando o botão "Executar".

Cada teste tem sua respectiva ficha e a navegação entre elas é feita por meio das setas da parte superior do formulário.

Um detalhe importante é a comunicação direta deste banco de dados com o sistema de manutenção. No mesmo ambiente, é possível abrir uma ordem de serviço, quando algum teste não estiver em conformidade, acessar o banco de peças da manutenção, relatórios de custos de manutenções, históricos da vida útil dos equipamentos e sua localização, entre outros.

Vale ressaltar que todo TCQ apresenta a identificação do equipamento, sendo, a cada teste realizado, gerado um histórico

\section{Programa de TCQ - HUCFF/UFRJ}

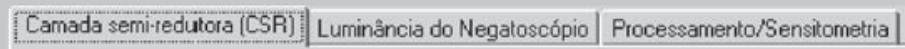

Objetivo: Avaliar se o feixe se raios $X$ atende ao requisito de filtração mínima.

Equipamentos: Câmara de ionização, suporte para a câmara de ionização, filtros de aluminio de $3 \mathrm{mmAl}$ [equipamento trifásicol ou 2,5mmAl [equipamento de 2 pulsos], fita

Procedimento: Selecionar $90 \mathrm{kVp}$ [yalor medido] ou próximo. Posicionar a câmara de ionização a $100 \mathrm{~cm}$ do foco $e$ a, pelo menos, $30 \mathrm{~cm}$ de espalhadores finclusive o tampo da mesal. Colimar o feixe de raios $X$ de forma a cobrir toda $\mathrm{a}$ área de medida da câmara. Selecionar de 10 a $50 \mathrm{mAs}$ e irradiar duas vezes. Colocar o filtro específico na saída do colimador e irradiar com a mesma técnica radiográfica duas vezes.

Análise do resultado: 0 valor encontrado deve ser de $2,5 \mathrm{mmAl}$ para aparelhos monofásicos ou de 3,0mmAl para aparelhos trifásicos.

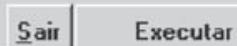

Figura 12. Protocolos dos TCQ. 
de TCQ de cada equipamento. Assim, o usuário pode ter acesso a todos os resultados dos testes de um determinado equipamento, acessando, no "menu", o item "Relatório" e a opção "Históricos dos TCQ”, conforme se vê na Figura 13. O usuário poderá escolher o TCQ que desejar gerar o relatório.

Na Figura 14 temos um exemplo de histórico gerado a partir da escolha acima.

Como o banco de dados pode ser utilizado em rede, é possível que vários usuários acessem e insiram informações, facilitando a comunicação entre o setor de manutenção e os responsáveis pela execução dos testes de constância.

O sistema também permite que a chefia do serviço de radiodiagnóstico possa acessar informações sobre os equipamentos, e acompanhar serviços, resultados dos testes e custos de maneira rápida e segura.

\section{CONCLUSÕES}

Pode-se concluir que elaborar um sistema de gerenciamento dos TCQ é tarefa plenamente exequiível, mesmo em serviço com a complexidade do HUCFF-UFRJ. O acesso às informações se dá de maneira bastante rápida e organizada, onde recuperar uma informação de testes anteriores é tarefa bastante simples.

$\mathrm{O}$ fato de a administração e a chefia do serviço poderem acessar as informações é um fator importante, já que é possível verificar o desempenho de todos os equipamentos de raios $\mathrm{X}$, podendo levar à tomada de decisões administrativas imediatas.

\section{Agradecimentos}

Os autores agradecem à Capes, pelo apoio financeiro.

\section{REFERÊNCIAS}

1. Brasil. Portaria 453. Diretrizes de proteção radiológica e radiodiagnóstico médico e odontológico. Brasília: Secretaria de Vigilância Sanitária, Ministério da Saúde, 1998.

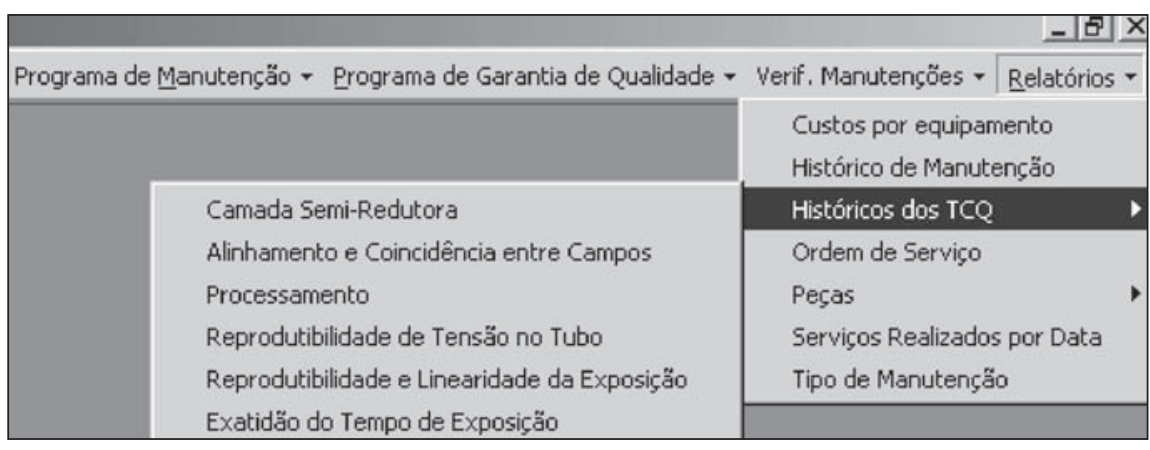

Figura 13. Item Históricos dos TCQ.

\section{Hospital Universitário Clementino Fraga Filho - UFRJ Serviço de Radiodiagnóstico}

Histórico dos Testes de Coincidência entre Campos por equipamento có digo:

\section{data do teste:}

Observacöes:

Figura 14. Histórico dos TCQ.

2. Bushong SC. Radiologic science for technologists: physics, biology and protection. 5th ed. St. Louis, Mosby, 1993.

3. Fonseca MM. Programa para avaliação e controle de serviços de radiologia de pequeno e médio portes de acordo com as diretrizes de proteção radiológica do Ministério da Saúde. (Dissertação de Mestrado). Paraná: Cefet-PR, 1998.

4. Tilly Júnior JG. Avaliações de doses de radiação em pacientes submetidos a exames radiológicos convencionais. (Dissertação de Mestrado). São Paulo: Universidade de São Paulo, 1997

5. Pina DR, Ghilardi Netto T, Rocha SL, Bronchi MAC, Trad CS. Construção de um fantoma homogêneo para padronização de imagens radiográficas. Radiol Bras 2000;33:41-4

6. Ros RA, Caldas LVE. Controle de qualidade de medidores de tensão não-invasivos em equipamentos de raios X, nível diagnóstico. Radiol Bras 2001; 34:89-94.

7. Khoury HJ, Morais Jr JB, Hazin CA. Avaliação dos negatoscópios e das condições de visibilização da imagem radiográfica em clínicas e hospitais de Recife, PE. Radiol Bras 2000;33:37-9

8. Koch HA, Peixoto JE, Neves ALE. Análise da infra-estrutura para a mamografia no Brasil. Radiol Bras 2000;33:23-9.
9. Arenson RL. Teaching with computers. Radiol Clin North Am 1986;24:97-103.

10. Koch HA, Pereira AA. Uma contribuição ao processo ensino-aprendizagem da radiologia. Radiol Bras 1988;21:85-91.

11. Marques PMA, Santos AC, Elias Júnior J, Goes WM, Castro CR, Trad CS. Implantação de um sistema de informação em radiologia em hospital universitário. Radiol Bras 2000;33:155-60.

12. Reiner BI, Siegel EL, Flage C, Hooper FJ, Cox RE, Scanlon M. Effect of filmless imaging on the utilization of radiology services. Radiology 2000;215: 163-7.

13. Reiner BI, Siegel EL, Hooper FJ, Glasser D. Effect of film-based versus filmless operation of the productivity of CT technologists. Radiology 1998 ; 207:481-5.

14. Geraldeli FE, Carvalho ACP, Koch HA, Azevedo ACP. Produção de material instrucional para o ensino da radiologia por meio da digitalização de imagens. Radiol Bras 2002;35:27-30.

15. Oliveira SR, Azevedo ACP, Carvalho ACP. Elaboração de um programa de monitoração ocupacional em radiologia para o Hospital Universitário Clementino Fraga Filho. Radiol Bras 2003;36:2734 